Published in Journal of Semantics: Bale, A. \& Barner, D. (2009). The interpretation of functional heads: Using comparatives to explore the mass/count distinction. Journal of Semantics, 26, 217252.

Please Note: This manuscript is posted for noncommercial use. It is the pre-publication version and, as such, may contain errors that are not present in the final publication.

\title{
The Interpretation of Functional Heads: Using Comparatives to Explore the Mass/Count Distinction
}

\author{
Alan Bale (Concordia University) \\ David Barner (University of California, San Diego)
}

\begin{abstract}
Comparative judgments for mass and count nouns yield two generalizations. First, all words that can be used in both mass and count syntax (e.g., rock, string, apple, water) always denote individuals when used in count syntax but never when used in mass syntax (e.g. too many rocks vs. too much rock). Second, some mass nouns denote individuals (e.g., furniture) while others do not (e.g., water). In this article, we show that no current theory of mass-count semantics can capture these two facts and argue for an alternative theory that can. We propose that lexical roots are not specified as mass or count. Rather, a root becomes a mass noun or count noun by combining with a functional head. Some roots have denotations with individuals while others do not. The count head is interpreted as a function that maps denotations without individuals to those with individuals. The mass head is interpreted as an identity function making the interpretation of a mass noun equivalent to the interpretation of the root. As a result, all count nouns have individuals in their denotation, whereas mass counterparts of count nouns do not. Also, some roots that have individuals in their denotations can be used as mass nouns to denote individuals.
\end{abstract}

\section{Introduction}

In this paper we seek to answer two questions. First, do functional heads that determine phrasal categories have a semantic interpretation? Specifically, in theories where lexical items are underspecified for syntactic categories such as mass and count, do nominal functional heads have an interpretation that is separate from lexical items? Second, what is the semantic nature of the mass-count distinction and what mechanisms are needed to explain this distinction? To answer these questions, we propose that there are two types of functional heads in noun phrases: 
one mass and the other count. These functional heads have interpretations that are independent of the lexical items with which they combine: both are interpreted as functions from sets of aggregates/groups to sets of aggregates/groups. The mass noun head is interpreted as the identity function, simply passing up the interpretation of the lexical root to the entire noun phrase. In contrast, the count noun head is a function from sets of aggregates that do not have individuals as minimal parts to sets of aggregates that do. The semantic nature of these functional heads accounts for two generalizations. The first generalization is that no mass nouns that have an associated count-noun usage ever allow for a comparison by number. This is best demonstrated by flexible nouns such as string and rock. There are clear differences in how we evaluate sentences such as those in (1a) compared to those in (1b).
(1) a. i. Seymour has more strings than Esme.
ii. Seymour has more rocks than Esme.
b. i. Seymour has more string than Esme.
ii. Seymour has more rock than Esme.

The sentences in (1a) are evaluated in terms of number whereas those in (1b) are evaluated in terms of length, weight or volume. Our interpretation of the count functional head as a function from sets of aggregates/groups without individuals to sets with individuals guarantees two things. First, all count nouns denote individuals (i.e., are associated with denotations that have individuals as minimal parts; see Section 5.2.1 for details). Second, no lexical items or roots that can be used in count noun phrases denote individuals. Hence, when such lexical items are used as mass nouns, the mass noun phrases also do not denote individuals. These two consequences of the interpretation of the count-noun head explain the generalization mentioned above.

The second generalization is that there are at least two types of mass nouns: those that allow for a comparison by number (such as furniture) and those that do not (such as water). This is demonstrated in (2).
(2)
a. Seymour has more furniture than Esme.
b. Seymour has more water than Esme. 
In (2a), truth conditions rely on a comparison by number (number of pieces of furniture) whereas in (2b) truth conditions rely on a comparison by weight or volume. In comparative constructions, mass nouns like furniture behave exactly like count nouns. Below, our treatment of the mass functional head as an identity function allows for these two classes to exist. As long as the lexical items can be interpreted as sets of aggregates with individuals or without, so too can mass nouns.

The outline of this paper is as follows. In Section 2, we briefly summarize current approaches to the semantic characterization of mass nouns and count nouns. In Section 3, we discuss different possible tests for evaluating the semantics of mass and count nouns, and argue that comparative constructions provide the most reliable test. In Section 4, we establish our two semantic generalizations with respect to mass and count nouns in greater detail. In Section 5, we present our proposal to account for these semantic generalizations, a proposal that crucially relies on providing an interpretation for nominal functional heads. In Section 5, we also provide a partial semantic analysis of comparative constructions such as those in (1) and (2), explaining how the nature of the noun denotation affects the truth values of the sentences.

\section{Theories of mass-count semantics}

In most discussions of the mass-count distinction, it is recognized that the use of a term in count syntax entails that at least part of its referent be discrete and/or bounded, and subject to being counted (Bloom, 1999; Bunt, 1985; Gillon, 1999; Gordon, 1985; Jackendoff, 1991; Link, 1983, 1998). The analysis of mass nouns, in contrast, has been the subject of much controversy. One of the most widely accepted proposals is that while count nouns always denote individuals, mass nouns never do (Bloom, 1994, 1999; Gordon, 1985; Link, 1983, 1998; Macnamara, 1986; Quine, 1960; Wisniewski, Imai, \& Casey, 1996). For example, the theories of Link (1983, 1998) propose join semi-lattices with minimal parts as the denotation of count nouns, and semi-lattices without minimal parts as the denotation of all mass nouns. As a result, mass nouns, unlike count nouns, do not denote individuals. According to psychologists such as Wisniewski, Imai, and Casey (1996:271), this difference means that speakers "conceptualize the referents of count nouns as distinct, countable, individuated things and those of mass nouns as non-distinct, uncountable, unindividuated things." 
As an alternative to this view of mass-count semantics, Gillon $(1992,1999)$ and Bunt (1985) argue that although count nouns denote individuals, mass nouns can be used to denote either individuals or non-individuals. Whether or not a particular mass noun can be used to denote individuals is not specified linguistically, but rather is determined via the speaker's inspection of the world. Thus, in the case of mass nouns like space, the speaker may encounter no units susceptible to enumeration and conclude that the term denotes continuous stuff (i.e., not individuals). In contrast, an inspection of the world may reveal that furniture is being used to talk about discrete bounded physical objects such as tables and chairs, and thus denotes individuals (see Gillon, 1999, for discussion of the similarities between his views and Bunt, 1985).

A third view extends these intuitions, and proposes that all mass nouns, and not just terms like furniture, have denotations with minimal parts and are inherently plural (Chierchia, 1998). ${ }^{1}$ According to this approach, differences between mass and count nouns exist, but not along the dimension of minimal parts. As Chierchia (1998:68) states, “...furniture is no less 'atomic' (i.e., made up of discrete sets of singularities) than piece of furniture or, indeed, table... At any rate, since in subdividing something we always get to an end, there is no principled reason to maintain that the denotation of mass nouns (even those whose granularity is unclear) do not have an atomic structure". Thus, things like furniture, mustard, and even fun can be divided up only to a certain point before there is no more furniture, no more mustard and no more fun. In each case, individuals are part of the denotation, though they may vary in how easily they are identified.

To summarize, we have reviewed three main alternatives to how the semantics of the mass-count distinction might be represented. Mass nouns may (1) have denotations containing only non-individuals, (2) be unspecified with regards to individuation, or (3) only have denotations containing individuals.

\section{How to evaluate the different theories of mass nouns}

Given the three alternatives discussed in Section 2, the question arises: what empirical facts can differentiate the three views? How can we tell whether mass nouns denote individuals? Below

\footnotetext{
${ }^{1}$ Chierchia only has semi-lattices with minimal parts. There are no continuous lattices. Also, he associates the entire semi-lattice with mass nouns and the entire semi-lattice minus the minimal parts with plural count nouns. However, he employs an operator that chooses the supremum of these lattices, thus making his theory essentially equivalent to Link's and Gillon's.
} 
we review three methods that have been discussed in the mass-count literature: (1) countability, (2) predicates and distributivity, and (3) comparative constructions. To inform the question of mass-count interpretation, a good method should yield reliable results that are independent of other grammatical factors. Two of the methods fail to meet this criterion, and are confounded by other grammatical properties.

\subsection{Why counting is unreliable for determining atomicity}

Countability, at first pass, sheds light on the relationship between nouns and individuals. Consider the sentence-template in (3), in which both mass and count nouns are syntactically possible replacements for $\mathrm{X}^{2}$

\section{(3) Seymour counted the X.}

With respect to this template, a possible generalization can be stated as follows: If a mass noun can be used in such a sentence felicitously, replacing $X$, then its denotation contains individuals. If not, then its denotation does not contain individuals. Underlying this is the assumption that counting requires that the noun denote individuals. On the surface, classifying nouns in this way is productive. For instance, it classifies all count nouns correctly: every count noun can be used felicitously in place of the X. Also, there are mass nouns that cannot be used felicitously in such a sentence. For example consider the potential sentences in (4).

(4) \#Seymour counted the anger/judgment/tolerance.

Each potential sentence is odd since it is difficult to image how one could count anger, judgment or tolerance.

However, a problem arises with respect to how counting is related to contextual factors independent of the mass-count distinction. Consider the sentence in (5).

\footnotetext{
${ }^{2}$ Alternative templates that explicitly involved a numeral such as There is/are $N X$, are not informative since they can only be used with count nouns. To adequately evaluate all theories, a syntactically neutral template is needed, since it is possible that numerals only select for count nouns due to their syntactic selectional restrictions rather than semantic requirements (see Gillon et al., 1999, for evidence supporting this possibility).
} 
(5) Seymour counted the sugar but not the water.

This sentence seems unacceptable in some contexts and acceptable in others. For example, the sentence is odd in a context in which there is a pitcher of water and a pile of sugar on the table. However it is more acceptable in a restaurant where there are bottles of water and packets of sugar on the table. One can talk about "counting sugar and water" when one counts sugar packets and bottles of water. There are two possible explanations of why the acceptability of the sentence changes with the context. First, it is possible that the type of nominal denotation changes as the context of use changes: ${ }^{3}$ the denotation of sugar might be continuous in one situation but have minimal parts in another. However, it is also possible that the denotation remains constant and that the circumstances of evaluation determine whether or not counting is permitted (i.e., evaluating the sentence requires counting, and therefore a prior division of the things/stuff into units in the situation). In this case, the nature the nominal denotation might be irrelevant in deciding whether or not counting is appropriate or acceptable. If so, such sentences would be unhelpful in determining whether nouns do indeed denote individuals.

There is some data that supports the second explanation. By all accounts, count nouns should specify stable units of individuation. However, consider the sentence in (6), when uttered in a factory where there is a conveyerbelt with boxes of pens, boxes of markrs and boxes of crayons passing by.

(6) Seymour counted the pens / markers / crayons.

The sentence in (6) usually refers to counting the individuals that make up the minimal parts of the denotation of pens, markers, and crayons. However, in a factory context where perhaps it is Seymour's job to keep tract of the number of boxes of writing implements, the relevant units for counting are boxes of pens, boxes of markers, and boxes of crayons. In this case, it is doubtful that the nature of the nominal denotation is changing in this context. The minimal parts of the count noun pens are always the individual pens in a given context. What is more likely is that the

\footnotetext{
${ }^{3}$ This position is similar to Bunt's (1985) proposal that the primary denotations of mass nouns do not contained atoms, yet in context of use, such denotations can be mapped to (secondary) denotations that do contain atoms.
} 
circumstances of evaluation select units for counting - i.e., boxes on a conveyorbelt.

In summary, the possibility that counting is influenced by the circumstances of evaluation make this way of classifying nouns less than ideal.

\subsection{Distributivity is an unreliable test for atomicity}

Another possible way of determining whether a denotation denotes individuals is to observe how a predicate can be distributed over its subparts. For example, the sentence in (7) has two prominent readings.

(7) The chairs in this room weigh 50 pounds.

This sentence can mean that all the chairs taken together weigh 50 pounds or that each chair weighs 50 pounds. By the second reading the predicate is distributed over individual chairs. Linguists like Bunt (1985) use such facts to argue that in a given context of use, certain nouns denote individuals. This line of reasoning rests on the assumption that if a noun supports distributivity in a given context, then it must denote individuals in that context. However, other evidence suggests that this assumption is not warranted. Distributivity of a predicate over an NP may have more to do with the predicate than the inherent divided reference of the noun (see Langendoen, 1978, Gillon, 1992, and Schwarzschild, 1996, for examples).

Consider the sentences in (8).
a. These spoons are priced at 100 dollars.
b. This cutlery is priced at 100 dollars.

What one receives for 100 dollars depends on the context of use. For example, either sentence could refer to a bunch of spoons that costs 100 dollars altogether. Alternatively, the sentences could refer to a stack of spoon-sets, each set in its own box and each box priced at 100 dollars. The sentences could even refer to individual spoons that cost 100 dollars each. The way that a predicate applies to its subject depends crucially on the context in which it is uttered, and not on whether the noun in question - i.e., spoon or cutlery - denotes individuals. 
It is possible that these different readings arise due to the denotation of the noun changing with the context. However, it is also possible that it is the predicate, or at least the way in which the predicate combines with the noun, that changes with the context, while the denotation of the noun remains constant. In other words, a predicate can be applied to the largest group in the denotation or to each member of a set of smaller subgroups, regardless of whether the denotation of the noun contains individuals. As a result, the subgroups over which the predicate is distributed need not be identical to the individuals in the noun's denotation. By this analysis (outlined in Langendoen, 1978, Gillon, 1992, and Schwarzschild, 1996) predicate distribution is an unreliable test for evaluating whether a noun denotes individuals. For example, consider how Bunt (1985) treats the phrase that sugar in (9).

(9) That sugar is cubical.

According to Bunt (1985:175), since (9) is true when referring to cubic lumps of sugar in a bowl on the table, as opposed to the ungranulated mass, the phrase that sugar in such a context should behave like the phrase those sugar lumps. In other words, Bunt's suggestion is that (9) demonstrates that the denotation of the NP in this situation has individuals as minimal parts. However, Bunt does not consider that (9) might only demonstrate a predicate's ability to distribute over subgroups in the subject's denotation: subgroups of the sugar that constitute the lumps. As noted with regards to (8), these subgroups need not be the same as the individuals that constitute the minimal parts in the noun's denotation. ${ }^{4}$ Since it is difficult to tell whether the predicate distributes over minimal parts or over higher subgroups, this method of classifying nouns is unreliable.

\subsection{Comparatives and noun phrases}

\footnotetext{
${ }^{4}$ An explanation of these phenomena that appeals to predicate-distribution seems necessary when comparative constructions involving the same nouns are involved. Consider the sentence in (i):

(i) a. John has more sugar than the Queen of England.

b. John has more sugar lumps than the Queen of England.

In a context in which John has several small lumps of sugar, and the Queen of England has one huge, giant lump of sugar sentence (a) is false whereas (b) is true. This is because sugar fails to provide the same default dimension for measuring that sugar lumps does: number of individuals. In other words, sugar does not alone (or even in its context of use) provide criteria for identifying individuals, and thus for using number as a measuring dimension. As demonstrated in (i) comparative constructions distinguish between an NP's ability to quantify over individuals and a predicate's ability to distribute over an NP, whereas predication tests alone cannot.
} 
Another method for exploring individuation involves comparative constructions. An interesting fact about such constructions is that the dimension of comparison implicated by a construction changes according to the denotation of the noun phrase. Consider the the sentences in (10).

(10) a. Esme has more chairs than Seymour has tables.

b. Esme has more water than Seymour has juice.

c. Esme has more rope than Seymour has string.

d. Esme has more gold in her ring than Seymour has silver in his necklace.

e. Esme has more anger than Seymour has sympathy.

In (10), the objects of comparison can be determined transparently for each sentence. In (10a), a measurement of Esme's chairs is compared to a measurement of Seymour's tables. In (10b) a measurement of Esme's water is compared to a measurement of Seymour's juice. And so on. In each case, the dimension of measurement shifts with the noun phrases. Specifically, the sentences in (10) can be evaluated on the basis of number, mass or volume, length, purity of materials, and emotional intensity, respectively. Given the sensitivity of the comparative construction to the noun, it becomes possible to classify nouns in terms of the selected dimension of comparison, beginning with a distinction between nouns that permit comparison by number and those that do not.

This method of classifying nouns is a highly reliable. First, it systematically identifies number as the default measuring dimension for count nouns, as in (11).

(11) a. Seymour has more apples than Esme.

b. Esme has more parties than Seymour.

c. Esme has more ideas than Seymour.

Each sentence is evaluated on the basis of number, as opposed to other dimensions of measurement such as mass or volume. For example, sentence (11a) is true in the context in which Seymour has three apples and Esme has one, even if the combined weight or volume of his three (tiny) apples is less than the weight or volume of her one (gigantic) apple. The same is 
true for the sentences in (11b) and (11c), where the person with the greater number of individuals is judged to have more $x$, where $x$ stands in for either apples, parties, or ideas.

Furthermore, this method reveals differences between mass and count nouns, demonstrating the sensitivity of these constructions to the mass-count distinction. Consider the mass noun usages in (12).

(12) a. Seymour has more water than Esme.

b. Esme has more glue than Seymour.

c. Seymour has more energy than Esme.

To evaluate these sentences, what matters is a continuous dimension of measurement, rather than number. Thus, even in a situation in which Esme has three portions of water and Seymour has only one portion, Seymour has more water so long as his single portion is greater overall in volume. The same results follow for sentences (12b) and (12c), where in each case a measurement cannot be based on number.

In most cases, the problems encountered by other methodologies do not affect the comparative method. For example, judgments elicited by comparative constructions are not affected by predicate distribution since comparisons do not involve dividing up a subject or object and applying it to a predicate. Also, comparative judgments are determined primarily by mass-count syntax even when the noun root and the context remain constant:

(13) a. Seymour has more rock / brick / string than Esme does.

b. Seymour has more rocks / bricks / strings than Esme does.

The sentences in (13a) and (13b) can be used in the same contexts, but result in different judgments. For example, consider a context where there are four small rocks that belong to Seymour and two very large rocks that belong to Esme. Despite the fact that there are salient individuals in this context - namely the rocks, the truth conditions of (13a) and (13b) differ. Only the count noun usages allow for a comparison by number even though individuals are 
contextually salient in both cases. ${ }^{5}$

Based on these observations, we submit that the comparative methodology offers the most reliable way of assessing mass-count semantics, since judgments are almost exclusively affected by alternations in mass-count syntax. In taking this view, we follow McCawley (1979), and psychologists such as Gathercole (1985), and Barner and colleagues (Barner \& Snedeker, 2005, 2006; Barner, Wagner \& Snedeker, 2008), who have each used comparative constructions to test the semantic distinction between mass and count nouns (see also Cresswell, 1976, who used comparatives to support his proposal that NPs are essentially measurement relations).

\section{Comparatives and the mass/count distintion}

\subsection{Data}

Previous investigations have used comparative constructions to evaluate the semantics of noun phrases. This paper adds two new generalizations to the literature. These generalizations, as we show, have profound consequences for the semantics of the mass-count distinction.

First, for common mass and count nouns, the comparative methodology yields results that are consistent with all existing theories of the mass-count distinction:

(14) a. Esme has more cups / plates / candles than Seymour.

b. Esme has more butter / water / toothpaste than Seymour.

The sentences in (14a) are true if Esme has a greater number of individual things (i.e., cups, plates, and candles) than Seymour, even if his things are bigger or heavier than Esme's. Since each noun is a count noun, number is specified as the dimension of comparison. In (14b) the sentences are true if Esme has a greater volume of stuff, even if the stuff is divided into discrete portions. Thus, if Esme has one large bottle of water and Seymour has three bottles, Esme still has more water than Seymour.

\footnotetext{
${ }^{5}$ Although context cannot force a comparison by number when the noun phrase does not permit such a comparison, the context can influence other types of measurement. For example, whether an object has more gold than another often depends on the context. Where purity matters, the comparison will be evaluated in terms of the percentage of gold in the object. However, where the overall amount of gold matters, the comparison will be evaluated in terms of mass.
} 
These intuitions can be extended to nouns that can appear in both mass and count contexts. For all such nouns, the count usage always specifies comparison by number, and the mass usage never does (i.e., some continuous dimension is selected):

(15) a. Esme has more ropes than Seymour.

b. Esme has more strings than Seymour.

c. Esme has more stones than Seymour.

(16) a. Esme has more rope than Seymour.

b. Esme has more string than Seymour.

c. Esme has more stone than Seymour.

In (15), Esme has more ropes, strings, or stones if she has a greater number of individuals. This is true regardless of how big Seymour's objects are. However, in (16), the basis for judgments is clearly different, and is consistently based on other dimensions of measurement such as length, mass, volume, or area. For example, (16a) is true if Esme has one ten-foot rope and Seymour has three one-foot ropes. In this same context, (15a) is false. The results of the comparative judgment shift systematically according to the syntactic status of the noun as mass or count, independent of the context, and despite the fact that perfectly good individuals are present in both cases (see Barner \& Snedeker, 2005, 2006, for evidence that adults, 3-year-olds and 4-year-olds shift quantity judgments according to mass-count syntax for terms like string and stone, and for novel mass and count nouns used to refer to discrete physical objects). Based on these examples and others like them (e.g., paper-papers, rock-rocks, tile-tiles, coffee-coffees, chocolate-chocolates, etc.) we propose Generalization 1:

(17) Generalization 1: No term that can be used in count syntax can also be used in a mass syntax to denote individuals.

It is important to note that this generalization cuts across subcategories of mass nouns and count nouns. Distributionally, nouns can be classified according to whether they are more amenable to count syntax (such as dog, cake, apple, and ideas), more amenable to mass syntax (such as water, mud, darkness, and coffee) or equally acceptable in either category (such as paper, rock, 
stone, thought and judgment). No matter the category preference, if a noun can appear in both categories it will denote individuals as a count noun but not as a mass noun. Thus, conceptual factors that might influence whether a noun is used as mass or count more frequently do not affect this generalization.

Also, Generalization 1 is true regardless of the type of semantic coercion that is associated with the noun's syntactic flexibility. It is true for so-called ground-noun coercion as in (18). In these cases, there is coercion from denotations containing individuals to denotations consisting of the stuff those individuals are made of. It is true of packaging coercion, as in (19). Here, there is coercion from substances to portions of substances. It is also true of individuationby-cause coercion, as in (20). In these examples, coercion is from denotations of emotions or mental states to denotations individuated in terms of the cause of those emotions or mental states. Finally, the generalization applies to individuation-by-instance coercion, as in (21), in which coercion is from nouns that denote a mental capacity or activity to nouns that denote instances of the capacity or activity in use.

(18) a. too many apples vs. too much apple

b. too many dogs vs. too much dog

c. too many bricks vs. too much brick

(19) a. too much water vs. too many waters

b. too much beer vs. too many beers

c. too much coffee vs. too many coffees

(20) a. too much anxiety vs. too many anxieties

b. too much fear vs. too many fears

c. too much hope vs. too many hopes

(21) a. too much thought vs. too many thoughts

b. too much judgment vs. too many judgments

c. too much kindness vs. too many kindnesses

Regardless of other idiosyncratic relationships between the interpretation of mass and count usages, wherever there is mass-count flexibility the count noun always denotes individuals whereas the mass noun never does. 
Taken in isolation, Generalization 1 seems to support theories of the mass-count distinction like that of Link (1983), which specify two distinct semantic domains for mass and count nouns, respectively. However, theories such as this cannot explain the data in (22):

(22) a. Esme has more furniture than Seymour.

b. Esme has more equipment than Seymour.

c. Esme has more footwear than Seymour.

Each of these comparative sentences contains a mass noun. However, unlike the terms in (14) or (16), these mass nouns support comparisons based on number. For example, (23a) can be paraphrased as Esme has more pieces of furniture than Seymour. Similarly, (14b) is equivalent to Seymour has more pieces of equipment than Esme, and (14c) to Esme has more shoes and boots than Seymour. ${ }^{6}$ Although such mass nouns are by no means in the majority, examples are not uncommon, and include luggage, underwear, company, glassware, wildlife, silverware, cutlery, mail, inventory, waterfowl, jewelry, and artillery, among others. In each case, the mass noun specifies comparison by number. These results lead us to propose our second generalization regarding mass-count semantics:

(23) Generalization 2: Some mass nouns (in the context of use) have individuals in their denotation, and others do not.

Mass nouns that can have individuals in their denotation like furniture and jewelry (heretofore “object-mass" nouns), differ from mass nouns like water or mustard, which never can, no matter

\footnotetext{
${ }^{6}$ According to McCawley (1979), mass nouns like furniture sometimes do not specify comparison by number. Instead, what is important is the extent to which the function of furnishing, for example, is satisfied. For example, he judges the sentence "Fred has more furniture than me" to be true in cases where I have a greater number of individuals but Fred has more types of furniture. However, type readings of this kind are accessible for most nouns, including count nouns. For example, McCawley notes that (ii) can be true if Fred has more types of clothes despite having fewer articles of clothing.

(ii) Fred has many more clothes than I do.

Furthermore, the same judgments hold for French where the translation of furniture is the count noun meuble.

(iii) Fred a plus de meubles que moi.

Based on these considerations, it seems that McCawley's examples highlight a type/individual ambiguity present in nouns like furniture (and indeed in most nouns), but that they do not provide evidence against the claim that certain mass nouns quantify over individuals (see Barner, Wagner \& Snedeker, 2008, for discussion).
} 
what the context or situation (see Barner \& Snedeker, 2005, and Barner, Wagner \& Snedeker, 2008, for psychological tests demonstrating this difference between object-mass nouns and mass nouns like mustard; see also Barner \& Snedeker, 2006, and Barner \& McKeown, 2005, for evidence regarding the origin of object-mass nouns in language acquisition). Paradoxically, the nouns that best denote individuals when used as mass nouns are the nouns that are most resistent to count syntax in English:
a. *Esme has some furnitures / equipments / footwears.
b. *Those furnitures / equipments / footwears belong to Esme.
c. * She has many furnitures / equipments / footwears.
d. * She has five furnitures / equipments / footwears

Together, the facts subsumed by Generalizations 1 and 2 are problematic for all three of the theories of mass-count semantics outlined above. They require a theory that allows mass nouns to denote individuals, but that prohibits them from doing so whenever the noun can also be used in count syntax. A corollary of this is that the theory must prevent mass nouns that denote individuals from also appearing in count constructions.

\subsection{Problems with alternative accounts}

\subsubsection{Uniform semantic domains for mass nouns}

The theories of Link (1983), Bloom (1994, 1999), and many others argue that mass nouns denote unindividuated quantities. While these accounts can explain the conversion facts, they do not predict the existence of object-mass nouns like furniture, which denote individuals. Thus, such theories can provide a natural explanation for Generalization 1 but are incapable of accounting for Generalization 2.

In response to this problem, some have suggested that although object-mass nouns name phenomena that are discrete individuals, they do not represent them as such, but instead lead speakers to construe their referents as unindividuated (e.g., Bloom, 1994; Wisniewski, Imai, \& Casey, 1996). According to Bloom (1994:45), "mappings relevant to the study of linguistic 
competence must be between grammatical classes and cognitive classes - not classes of entities in the world." While chairs and tables are individuals and count as instances of furniture, the word furniture does not pick them out as individuals, but leaves them unindividuated. ${ }^{7}$

Accepting this logic, we are still led to conclude that mass nouns like furniture denote individuals. Note that the comparative method does not lead to judgments based on number whenever discrete individuals in the world are named. As demonstrated in (15) and (16), the interpretation of nouns like string and stone shift as the nouns change between mass and count, even when the entities in the world remain constant (and clearly qualify as discrete individuals, as evidenced by their status as such when named by count nouns). Comparative judgments are sensitive to shifts in mass-count syntax and nonetheless indicate that at least some mass terms denote individuals.

\subsubsection{Inherent plurality}

Like Link (1983), Chierchia (1998) argues that all mass nouns share a common semantic domain. However in contrast to Link, Chierchia proposes that all mass nouns have denotations with minimal parts. As a result, his theory is unable to differentiate object-mass terms from other mass nouns.

Chierchia provides at least one mechanism by which his theory might account for different types of mass nouns. He suggests that mass nouns like water differ from those like furniture according to their relative vagueness. ${ }^{8,9}$ What counts as individuals for a particular mass noun on a given occasion is not specified linguistically but rather is determined contextually. The difference between object-mass nouns and other mass nouns is determined by how readily one can identify the individuals that make up the minimal parts. However, vagueness seems unable to account for Generalizations 1 and 2. As Chierchia notes, the effects of vagueness are normally mitigated by providing details in the context. In speaking about the sentences the rice is from

\footnotetext{
${ }^{7}$ Also supporting this view, Wisniewski et al. (1996:295) claim that:

...on a particular occasion, we may conceptualize a swan, several ducks, and a heron on a lake as an unindividuated group called waterfowl, and not think of them individually as birds.

${ }^{8}$ We assume that Chierchia is using the term "vague" in much the same sense as Quine (1960:126), where what counts as a minimal part is "left unsettled".

9 "What set of atoms generates the extension of a mass noun can be quite vague (though for mass nouns like furniture it isn't substantially more vague than for table) and typically varies from context to context." (Chierchia, 1998:70)
} 
Italy and the grains of rice are from Italy, Chierchia (1998:85) states, "what counts, in context, as minimal parts has to be made clear. In a situation where it is clear that only whole grains of rice count as rice then [the two sentences above] will have the same truth conditions". Yet no matter how explicit the context is for sentences like the one in (28), there can never be a comparison by number.

(28) Esme has more rice than Seymour.

The sentence in (28) is false so long as Seymour has more rice in mass or volume, no matter how much it is emphasized that Esme has more grains or bags. ${ }^{10}$

Vagueness also fails to make the correct prediction for words that vary in mass-count usage cross-linguistically. In French, one speaks of des épinards (roughly "some spinaches"), des cheveux ("some hairs" - even in the context of washing it/them), and des pâtes ("some pastas"). In each case, the French word is a count noun, but the equivalent English word is a mass noun. Using the comparative methodology, Inagaki and Barner (in press) showed that French speakers base comparative judgments for spinach, hair, toast, pasta, etc., on number, while English speakers base judgments on continuous dimensions like mass and volume. Thus, the sentences in (29a) lead to judgments based on total mass or volume, whereas those in (29b) lead to judgments based on number:

a. Esme has more spinach / hair / pasta than Seymour.

b. Esme a plus d'épinards / cheveux / pâtes que Seymour.

In English, if Esme has more individual leaves of spinach, this does not mean that she necessarily has more spinach than Seymour. Seymour could still have a greater mass or volume. However, the opposite is true in French (though mass or volume may act as a proxy for number when the leaves are not countable in a context). Such examples suggest that discrete individual things do exist in the world, and that these things can be encoded linguistically. However, no

\footnotetext{
${ }^{10}$ Another significant problem for Chierchia is that it is unclear why vagueness should systematically affect mass nouns but not count nouns. Many count nouns suffer greatly from vagueness. As Quine (1960:126) notes in his discussion of the count noun mountain, "it is not clear when to declare a saddle to be in the middle of one mountain and when between two mountains. The issue makes all the difference between one mountain and two." Nonetheless, unlike mass nouns it is never acceptable to base comparative judgments for a term like mountain on mass or volume.
} 
amount of contextual specification in English permits comparison by number in these cases. The semantic difference appears to be purely grammatical.

\subsubsection{Multiple domains for mass nouns}

Theories that support two or more semantic domains for mass nouns, such as Bunt (1985) and Gillon (1992, 1999) provide an account of the Generalization 2, but fail to account for the conversion facts as stated in Generalization 1. By claiming that the denotations of particular mass nouns are underspecified, both theories allow mass nouns to denote sets of "ensembles" (for Bunt) or "aggregates" (for Gillon) that may or may not be composed of individuals. ${ }^{11}$ According to these authors, the nature of a noun's denotation is determined via an inspection of the world: when discrete individuals are present the mass noun will denote individuals, but where no individuals exist it will not (see Gillon, 1999:9 and Bunt, 1985:130). Hence it is predicted that some nouns should denote individuals while others should not. This is the essential data point summarized in Generalization 2.

However, these proposals provide no account of why mass nouns like stone and string should fail to denote individuals as mass nouns but not as count nouns. They also fail to account for cross-linguistic variation such as the difference between French and English demonstrated in (29). In all such cases, discrete physical objects may exist in the world, but only the count usages lead to comparative judgments based on number. In general, these theories offer no account of the conversion facts of Generalization 1 or the observation that certain words denote individuals as count nouns but not as mass nouns despite the things in the world remaining constant.

\section{Interpreting functional heads}

The problem posed by Generalizations 1 and 2 is how to describe the lexical and syntactic sources of individuation. Generalization 1 requires an account of how count syntax specifies reference to individuals. In contrast, Generalization 2 requires an account of how some mass nouns can denote individuals while others do not. In this section, we provide an interpretation for

\footnotetext{
${ }^{11}$ In Bunt's (1985) theory, the denotation of a mass noun has minimal parts only when it is interpreted in the context of use. The primary denotations do not have minimal parts, but can be mapped to secondary denotations that do have minimal parts, given the appropriate context.
} 
the functional heads of count-noun and mass-noun phrases that accounts for these facts. We begin by outlining what we take to be the basic syntactic form of such phrases. We then proceed to outline a detailed functional interpretation of these forms, focusing on the interpretation of root lexical items and the functional heads that determine the category of the phrases. Next, we explain how these functional interpretations can be combined in comparative constructions to yield the data presented above. Our syntax and the general strategy of our semantic approach are similar to Borer (2005) and Krifka (1995), with important differences in the details.

\subsection{Syntactic assumptions}

\subsubsection{Features distinguishing mass from count}

Before providing an interpretation for mass and count NPs, let us first note our syntactic assumptions. ${ }^{12}$ Three facts are relevant to our analysis. First, certain quantifiers/determiners only select count nouns - e.g., many, several, a few, every, and the numerals two, three, four, etc. Second, certain quantifiers/determiners only select mass nouns - e.g., much and little. Third, quantifiers such as a lot of, this, the, all, and some can be used with either kind of noun. Minimally, a syntax for the mass-count distinction should account for these facts.

These facts can be explained by a framework that assumes that lexical items are unspecified for syntactic categories (a hypothesis first defended for a subset of lexical items by Chomsky, 1970; for an extension of this view, see Barner \& Bale, 2002, 2005; Borer, 2005; Halle \& Marantz, 1993; Harley \& Noyer, 1999; Marantz, 1997). By this approach, lexical items become nouns or verbs in the syntax by combining with nominal and verbal heads. Similarly, mass and count nouns can be distinguished syntactically by features of the functional head in which the noun appears. Minimally, two features are required. One feature, which we call ' $n$ ', determines whether or not the phrase is nominal. The second feature, which we call ' $c$ ', determines whether or not the phrase is count. When appearing together, these features are bundled under the same syntactic head.

\footnotetext{
${ }^{12}$ Our syntactic structures are similar to those proposed by Borer (2005). Only the labelling of categories is different. The quantifier facts that motivate our syntactic structures are also used to motivate Borer's structures. However, we will leave facts about classifiers in Mandarin Chinese out of the present discussion.
} 
This system permits two syntactic possibilities for noun phrases (Figure 1): one in which a root lexical item combines with just the nominal feature ' $n$ ' and another in which a root combines with ' $n$ ' and the count feature ' $c$ '. The example in (a) represents a count-noun functional head, the example in (b) a mass noun functional head.

a.

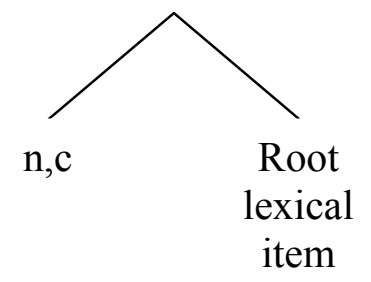

b.

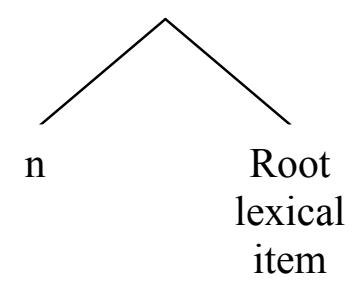

Figure 1: The tree in (a) shows the basic structure of a count noun phrase whereas the tree in (b) shows the basic structure of a mass noun phrase.

We represent lexical items (or roots) with the root symbol " $\sqrt{ }$ " preceding the canonical orthographic representation of the word (see Pesetsky, 1994, for a similar use of this symbol). Thus, the lexical item associated with furniture is $V_{\text {furniture }}$ and cat is $\sqrt{ }$ cat). The mass noun furniture and the count noun cat have the following syntactic structures.

a.

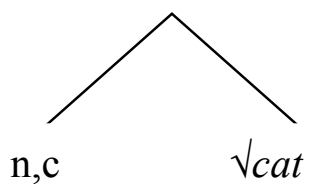

b.

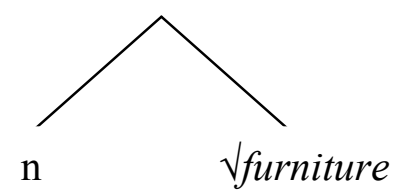

Figure 2: The tree in (a) shows the basic structure of the count noun phrase cat whereas the tree in (b) shows the basic structure of the mass noun phrase furniture.

By this account, the distribution of quantifiers/determiners can be easily explained. First, since all quantifiers/determiners apply to nouns, they all require that their complement have the feature ' $n$ '. Second, quantifiers/determiners that only apply to count noun phrases (such as several) require that their complement also have the feature ' $c$ '. Third, quantifiers/determiners that only apply to mass noun phrases (such as much) require that their complements do not have the feature ' $c$ '. Finally, quantifiers/determiners that apply to both count nouns and mass nouns (such 
as all) have no requirements or restrictions with regard to ' $c$ '. This exhausts all possible combinations of features.

\subsection{Interpretations}

With the syntactic forms specified in Section 5.1, we can now explore the interpretation of roots and functional heads. As outlined below, we propose that roots are interpreted as semi-lattices (sets of aggregates/pluralities). The mass noun functional head is interpreted as an identity function whereas the count noun functional head is interpreted as a function from semi-lattices without individuals to semi-lattices with individuals.

\subsubsection{The interpretation of roots.}

Following an influential tradition established by Link (1983), we adopt an algebraic semantics for NPs. Specifically we assume that roots are interpreted as join semi-lattices. Such structures have proven to be useful for providing a semantic analysis of how quantifiers and determiners interact with count and mass nouns while also accounting for their referential properties (see Bunt, 1985; Gillon, 1992, 1999; Chierchia, 1998, etc).

The join semi-lattices relevant to our semantics implicate sets of aggregates. Aggregates (sometimes also called pluralities, sums, groups, collections, or ensembles) can be quantities of individuals or of substances, energies, ideas etc. We will represent aggregates by using a sequence of lowercase letters (e.g., $a b c, b c, a, b$, etc.). As is commonly noted in the literature on aggregates, an ordering relation can be defined through a sum/join operator $(\oplus)$ that maps any two aggregates to a single aggregate that is a combination of the two (cf. Link 1983). ${ }^{13} 14$

Definition of $\oplus$ : For all aggregates $x$ and $y: x \oplus y=x y .{ }^{15}$

With this operator, one can define the aggregate-part-of relation, symbolized by ' $\leq$ '.

\footnotetext{
${ }^{13}$ Sets and aggregates are often interchangeable due to their similar structural properties: both can form join semilattices. Here, we prefer aggregates since their representation is more amenable to the representation of atomless semi-lattices, which might be crucial for interpreting a large class of mass nouns.

${ }^{14}$ Alternatively, a sum operator can be defined through a given ordering relation.

${ }^{15}$ Note that this operator is idempotent (for any aggregate $x, x \oplus x=x$ ), commutative (for all aggregates $x$ and $y, x \oplus$ $y=y \oplus x$ ), and associative (for all aggregates $x, y$, and $z,[(x \oplus y) \oplus z]=[x \oplus(y \oplus z)]$ ).
} 
Definition of $\leq$ : For all aggregates $x$ and $y, x \leq y$ if and only if $(x \oplus y)=y$.

Due to the properties of $\oplus$, the relation $\leq$ represents a partial order (that is to say, it is reflexive, anti-symmetric and transitive). ${ }^{16}$ For our semantics, the interpretation of every root $R$ is a set of aggregates $X$. Thus $\llbracket R \rrbracket=\mathrm{X}$. Furthermore, for all aggregates $x$ and $y$ that are members of $X$, their sum $x \oplus y$ is also a member of $X$. In other words, the interpretation of every root is closed under sum. Also, since the members of $X$ are partially ordered by $\leq$, the interpretation of each root is a (sum/join) semi-lattice.

There are three types of semi-lattices that are relevant for a discussion of mass and count nouns. In the first - a limited semi-lattice - every member of the semi-lattice is composed of minimal parts. A minimal part is defined as follows:

Definition of a minimal part: an aggregate $x$ is a minimal part for a set of aggregates $X$ iff $x \in X$ and for any aggregate $y$, such that $y \neq x$ and $y \in X$, it is not the case that $y \leq x$.

This definition of minimal parts might appear weaker than most, since by our account minimal parts are not necessarily atoms. Often it is assumed that that two distinct minimal parts cannot share aggregates. However, according to the definition above, two minimal parts could share aggregates. For example, consider the following set $X=\{a c, a d, b c, b d, a c d, a b c, a b d, b c d$, $a b c d\}$. The set $X$ is closed under sum and forms the lattice depicted in Figure 3.

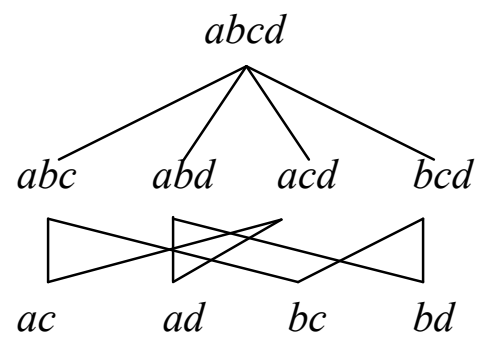

\footnotetext{
${ }^{16}$ It is simple to prove that $\leq$ is a partial order. Reflexivity: For all aggregates $x,(x \oplus x)=x$ and hence $x \leq x$. Antisymmetry: For all aggregates $x$ and $y$ such that $x \leq y$ and $y \leq x$, it follows that $x \oplus y=y$ and $y \oplus x=x$. By commutativity, $(y \oplus x)=y$ and hence by substitution $x=y$. Transitivity: For all aggregates $x, y$ and $z$, if $x \leq y$ and $y$ $\leq z$, then $x \oplus y=y$ and $y \oplus z=z$. Since $y \oplus z=z$, it follows that $(x \oplus y) \oplus z=z$ by substitution. By associativity, $(x$ $\oplus y) \oplus z=x \oplus(y \oplus z)$. By substitution, $x \oplus(y \oplus z)=x \oplus z$. Since $(x \oplus y) \oplus z=z$ it follows that $x \oplus z=z$. Hence, if $x \leq y$ and $y \leq z$, then $x \leq z$.
} 
Figure 3: The join semi-lattice $X=\{a c, a d, b c, b d, a b c, a b d, a c d, b c d, a b c d\}$. Note this lattice can be generated from the minimal parts $\{a c, a d, b c, b d\}$.

In this lattice the minimal parts share aggregates. For example, the aggregate $a$ is part of both $a c$ and $a d$. Similarly, $c$ is part of $a c$ and $b c$. This type of lattice can represent roots that denote aggregates with non-homogenous components, such as $\sqrt{ }$ succotash (an example from Sharvey, 1979). All succotash contains lima beans and corn, but it would be unusual to call a piece of corn succotash if it were not paired with at least one bean. Thus, the lattice in Figure 3 could represent an amount of succotash in which $a$ and $b$ are portions of beans and $c$ and $d$ are portions of corn. ${ }^{17}$

The second type of lattice relevant to our account is an individuated semi-lattice, which has individuals as minimal parts, where individual is defined as follows:

Definition of an individual: an aggregate $z$ is an individual for a set of aggregates $X$ iff $z$ is a minimal part for $X$ and for all aggregates $y \in X$, either (i) $z \leq y$ or (ii) there is no $w \leq$ $z$, such that $w \leq y$.

This definition guarantees that an individuated semi-lattice will not have minimal parts that share an aggregate. An example of an individuated semi-lattice is given in Figure 4.

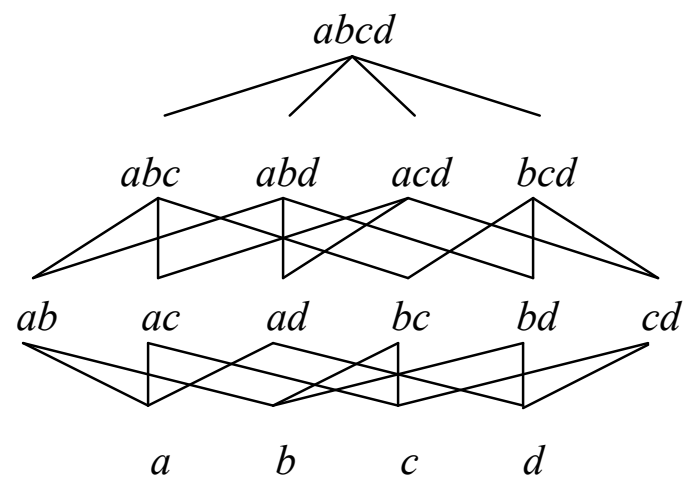

Figure 4: The semi-lattice $X=\{a, b, c, d, a b, a c, a d, b c, b d, c d, a b c, a b d, a c d, b c d, a b c d\}$. Note, this lattice can be generated from the set of individuals $\{a, b, c, d\}$.

\footnotetext{
${ }^{17}$ We use succotash as a toy example to illustrate our point. We are simplifying greatly here, however from our representation one is able to get a general outline of the type of denotation that we have in mind.
} 
In this structure, no part of a minimal part can appear in another aggregate without all of its parts appearing in that aggregate. The notation in Figure 4 makes this point seem trivial, since the minimal parts are represented by single letters (e.g., $a, b, c$, or $d$ ). However, we can replace the aggregates a, b, c, and d with complex aggregates such as ef, $g h, i j$, and $k l$ without changing the classification of the minimal parts as individuals. An example is shown in Figure 5.

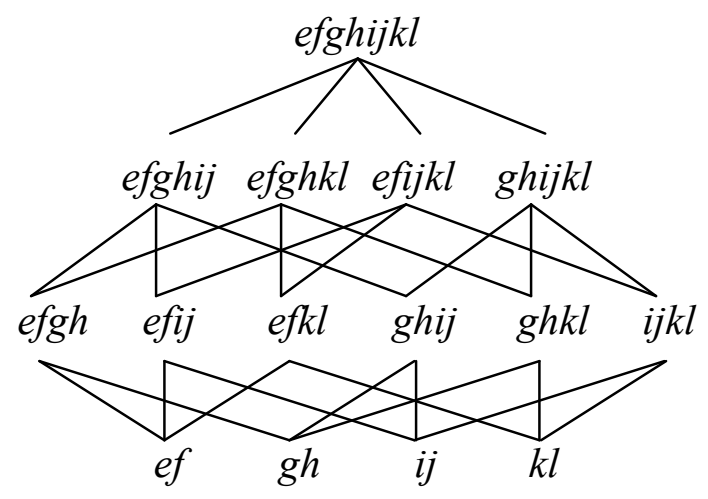

Figure 5: The lattice $X=\{e f, g h, i j, k l$, efgh, efij, efkl, ghij, ghkl, ijkl, efghij, efghkl, efijkl, ghijkl, $e f g h i j k l\}$. Note, this lattice is can be generated from the set of individuals $\{e f, g h, i j, k l\}$.

The important point is that the relation of the minimal parts to other members of the lattice defines what counts as an individual in the lattice. For example, since ef is a minimal part in Figure 5, and since no subpart of ef appears in any other aggregate without all of ef appearing in that aggregate, the aggregate ef is an individual in the lattice. These individuated semi-lattices can be used to represent denotations that contain individuals, as is required for object-mass nouns like furniture.

Unlike limited and individuated semi-lattices, the third type of semi-lattice has no minimal parts and hence no individuals either. We call this semi-lattice a continuous semi-lattice. Unlike the others, these lattices cannot be easily represented graphically or symbolically. However, they are nonetheless well-defined mathematical objects. A set of aggregates $X$ is a continuous semi-lattice iff $X$ is closed under sum and $X$ has no minimal parts. In other words, for every aggregate $x$ that is a member of the lattice $X$, there is another aggregate $y$ in $X$ such that $y \leq$ $x$ and $y \neq x$. Intuitively, this means that for any aggregate in $X$ there is an even smaller part of that aggregate that is also in $X$. Continuous semi-lattices can be used to represent lexical roots such as space, whose denotations might not contain minimal parts. 
Whether continuous semi-lattices are needed (as suggested by Link, 1983) or whether every lattice must have minimal parts (as suggested by Chierchia, 1998) is not crucial to our remaining discussion, nor for our proposal regarding the role of functional heads in NP interpretation. What does matter is that there is a difference between individuated lattices on the one hand and non-individuated lattices on the other (whether 'continuous' or simply 'limited').

\subsubsection{The interpretation of functional heads}

There are two functional heads that require an interpretation: the mass noun head represented by the feature ' $n$ ' and the count noun head represented by the bundle of features ' $n, c$ '. By our account, the mass noun head is interpreted via an identity function. As a consequence, the interpretation of mass nouns is identical to the interpretation of the root in isolation.

Interpretation of the mass noun functional head: $\llbracket n \rrbracket=i$, where $i$ maps every set onto itself.

Determiners such as the and that (among others) operate on the unmodified semi-lattice that represents the denotation assigned to the root. For instance, as suggested by Link (1983), the can be represented as a (partial) function from semi-lattices to the supremums of the semi-lattices (the largest and top-most aggregate in the lattices). This function is partial since it requires that each semi-lattice in its domain have a unique largest and top-most aggregate. As discussed in Link (1983) this representation predicts both the determiner's presuppositions and truth conditions. As another example, the interpretation of the determiner that can be interpreted as a function from semi-lattices to a member of that lattice - in effect, a choice function that is sensitive to contextual cues such as proximity, pointing, and topics in the previous discourse.

For clarity, consider an example. Suppose we are in a room with two tubs of water and several pieces of furniture (a few chairs, a table, and a sofa). The denotation of the root $\bigvee_{\text {water }}$ would be the set of all the aggregates of water (all the parts of water), called $W$. This denotation might be a limited semi-lattice or a continuous semi-lattice. Whatever the choice, the denotation will have a top-most member (all the water in both tubs taken together). Call this member $a$. Also, the denotation will have as separate members all the water in one tub (call it $b$ ) and all the 
water in the other tub (call it $c$ ). Suppose also that $b$ is contextually emphasized (perhaps by someone pointing to it). Note that in this context the supremum $a$ is identical to the aggregate $b c$. In this situation, the phrases the water and that water have the denotations in Figure 6.

a.
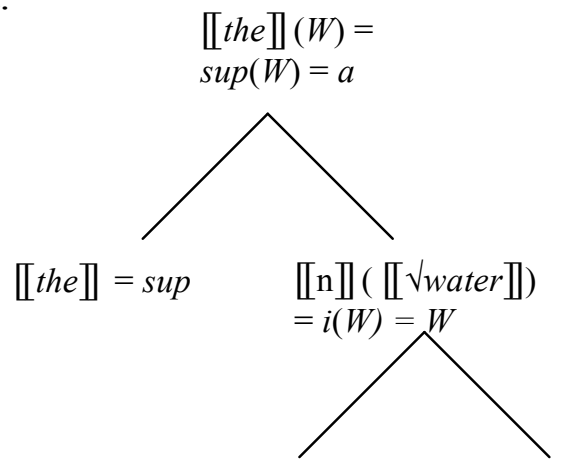

$\llbracket \mathrm{n} \rrbracket=i \quad \llbracket \sqrt{ }$ water $\rrbracket=W$ b.

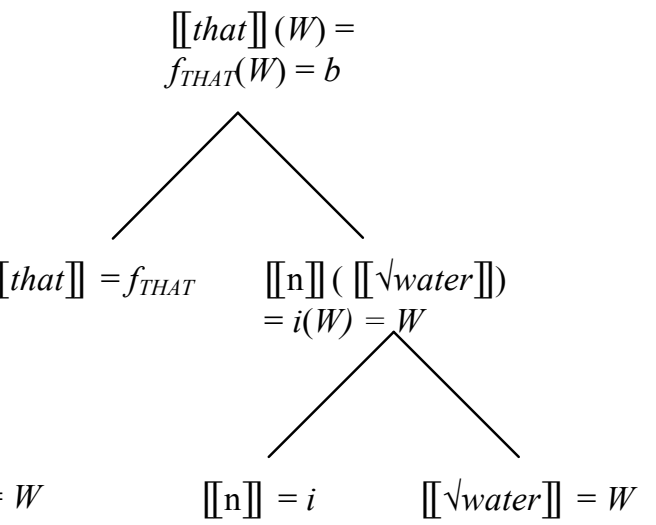

Figure 6: The root $\sqrt{ }$ water denotes the semi-lattice $W$ whose top-most member is $a$. The lattice also contains contextually prominent aggregates $b$ and $c$, where $b$ is farther away than $c$. The function $f_{T H A T}$ selects from any given set the contextually prominent aggregate. (a) shows the compositional meaning of determiner phrase the water. (b) shows the compositional meaning of the determiner phrase that water.

In the same context, the denotation of $\sqrt{ }$ furniture would be the set of furniture and furniture pluralities. Thus if $a, b$, and $c$ were the chairs, $s$ the sofa, and $t$ the table, then the denotation would be the set $F=\{a, b, c, s, t, a b, a c, a s, a t, b c, b s, b t, c s, c t, s t, a b c, a b s, a b t, a c s, a c t$, ast, $b c s, b c t, b s t, c s t, a b c s, a b c t, a b s t, a c s t, b c s t, a b c s t\}$. This is an individuated semi-lattice. Given this denotation, the phrases the furniture and that furniture have the interpretation in Figure 7.

a.

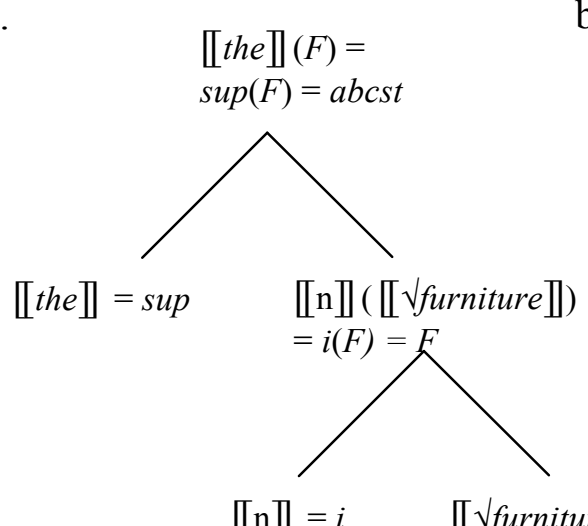

b.

$\llbracket$ that $\rrbracket(F)=$

$$
f_{\text {THAT }}(F)=a b c
$$

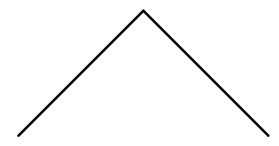

$\llbracket$ that $\rrbracket=f_{\text {THAT }}$

$\llbracket \mathrm{n} \rrbracket(\llbracket \sqrt{ }$ furniture $\rrbracket)$

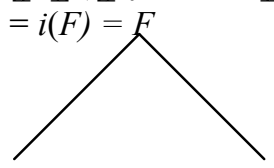

$\llbracket \mathrm{n}]]=i \quad \llbracket \sqrt{ }$ furniture $\rrbracket=F$

Figure 7: The root Vfurniture denotes the semi-lattice $F=\{a, b, c, s, t, a b, a c, a s, a t, b c, b s, b t, c s$, $c t, s t, a b c, a b s, a b t, a c s, a c t, a s t, b c s, b c t, b s t, c s t, a b c s, a b c t, a b s t, a c s t, b c s t, a b c s t\}$, whose topmost member is $a b c s t$. The lattice also contains the contextually prominent aggregate $a b c$ that is 
some distance from the speaker. The function $f_{T H A T}$ selects from any given set the contextually prominent aggregate that is further away from the speaker. (a) shows the compositional meaning of determiner phrase the furniture. (b) shows the compositional meaning of the determiner phrase that furniture.

In summary, mass functional heads do not alter the interpretation of root lexical items. If a root is interpreted as an individuated lattice then the mass NP is interpreted as an individuated lattice. ${ }^{18}$ If the root represents an un-individuated lattice then the mass NP does too.

The interpretation of the count noun head differs substantially from that of the mass noun head. It is not interpreted as an identity function but rather as a function that alters the type of semi-lattice it takes as an argument. Furthermore, it is only a partial function. Its domain consists only of semi-lattices that are non-individuated (continuous or limited) semi-lattices. This partial function maps these types of lattices to individuated semi-lattices.

(Underspecified) interpretation of count noun functional head: $\llbracket n, c \rrbracket=I N D$, where $I N D$ is a function from semi-lattices to semi-lattices. The domain of $I N D$ does not include any individuated semi-lattices. The range of IND only includes individuated semi-lattices.

Here, we set aside the details of how each lexical denotation is mapped to an individuated semilattice. For the present purposes, the important point is that there is a systematic relationship between mass and count usages of single lexical roots, like apple, fear, tile, and rock. The count usages always denote individuals, whereas the mass usages do not. This observation suggests that each lexical root supports both kinds of interpretation, but is semantically underspecified, and requires mass-count syntax (i.e., IND) to select between interpretations. For example, by one account concepts like apple supply both a principle of application (which distinguishes applestuff from orange-stuff or banana-stuff) but also a principle of individuation (which indicates how much of this stuff, and in what arrangement, counts as one individual, or as two). The relationship between mass and count interpretations may depend on such conceptual factors, and thus the difference between them may vary idiosyncratically from one mass-count pair to another (see Barner \& Bale, 2002, 2005, for details). For example, the relation between the mass and

\footnotetext{
${ }^{18}$ In this way our theory differs from those of Sharvey (1979) and Borer (2005) who maintain that count syntax introduces a hidden classifier not present in mass syntax. Their theories provide no way of deriving a number interpretation for mass nouns like furniture. Our theory can be considered a simple expansion or addition onto their theories to account for the empirical facts discussed in this paper.
} 
count interpretations of water is quite different from the relation between those for chicken (the first can be called a "packaging" relation, whereas the second a "grinding" relation). Amidst these interesting idiosyncrasies, however, there is a systematic generalization, which is the focus of our discussion: IND maps lexical items that denote only non-individuated semi-lattices to denotations of individuated semi-lattices (i.e., intensions that denote only non-individuated semilattices to intensions that denote only individuated semi-lattices; for simplicity we leave out the intensional talk). We assume that idiosyncrasies specified in the lexical concept help determine this mapping.

By this proposal, all count nouns are derived from lexical items that denote nonindividuated semi-lattices. Relevant to this hypothesis is the fact that lexical items differ with respect to categorical preference (although most lexical items can appear in either category). Some items are more natural as mass nouns (e.g., water), some are more natural as count nouns (e.g., apple), while others seem fully flexible (e.g., tile, rock, stone, and judgment). In our view, these differences in "preference" cannot be explained by a grammatical system. Grammar can explain the possibility of flexible use, but not the frequency with which forms actually occur.

In a previous set of papers (Barner \& Bale, 2002, 2005), we discussed this issue with respect to noun-verb flexibility in detail. Our main observation was that, given the capacity to generate novel nouns and verbs from words first heard as members of the opposite category, any grammar must account for noun-verb flexibility via grammatical rules. However, by all accounts, such rules cannot explain why one lexical item should appear more frequently (or seem more natural) as a noun or as a verb. The grammar specifies that both options are available without specifying a preference. The same is true for the mass-count distinction. Given the capacity to produce novel mass and count nouns, the grammar must include rules that generate flexibility, while allowing for differences in the frequency of mass and count usage across items. In our discussions of noun-verb flexibility, we suggested that such differences in usage may stem, in part, from extra-grammatical factors. For example, some possible uses may be blocked by existing vocabulary items, as noted by Clark (1993). Other possible uses may be pre-empted by potentially ambiguous homophony (see Bauer, 1973; Clark, 1982, 1993; Rainer, 1988, for discussion). Furthermore, Dressler and Ladanyi (2000) list a number of additional factors that likely contribute to restricting grammatical productivity, including pragmatic incompatibilities related to reference (Bauer, 1983; Corbin, 1997), pragmatic self-evidence (e.g., bearded man 
versus *eyed man), stylistic convention, and various socio-linguistic considerations. In short, we believe that the use to which words are put, and the availability of contexts for their interpretation, goes beyond the types of generalizations that we discuss here, and probably beyond the scope of a theory of grammar.

Before discussing some examples of our interpretation of the functional heads, we would like to clarify that we are not suggesting that the mass noun interpretation is conceptually more basic than the count noun interpretation. The functional interpretation of the count noun head simply represents a mapping involving two different denotations. It does not represent a derivation of count-like concepts from mass-like concepts. The relation between mass and count concepts is a separate matter altogether which has little to do with the syntactic representation of nominal categories and their interpretations.

For clarity, consider an example. Suppose we are in a room filled with rocks and apples. Suppose $W$ is the denotation of the root $\sqrt{ }$ rock and that $Z$ is the denotation of the root $\sqrt{ }$ apple. Thus, $W$ contains any aggregate that consists only of rock-stuff and $Z$ contains any aggregate that consist of apple-stuff. We assume that both $W$ and $Z$ are non-individuated semi-lattices. In this context, certain amounts of rock-stuff form objects (that is, they form rocks). Let's represent the set of these objects as $X=\{a, b, c\}$. The sub-lattice that has these objects as atoms would be the set $X^{\prime}=\{a, b, c, a b, a c, b c, a b c\}$. Also in this context, certain amounts of apple-stuff (arranged in the appropriate way according to how apples are individuated) form objects (that is, they form apples). Let's represent the set of these objects as $Y=\{m, n, o\}$. The sub-lattice that has these objects as atoms would be the set $Y^{\prime}=\{n, m, o, n m, n o, m o, n m o\}$. Given this context, the interpretation of the noun phrases the rocks and the apples will be as in Figure 8.

a.

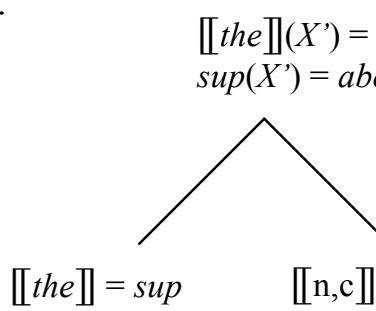

$\llbracket$ the $\rrbracket=\sup \quad \llbracket \mathrm{n}, \mathrm{c} \rrbracket(\llbracket \sqrt{ }$ rock $\rrbracket)$

$=I N D(W)=X^{\prime}$

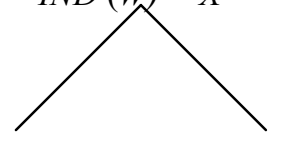

$\llbracket \mathrm{n}, \mathrm{c} \rrbracket=I N D \quad \llbracket v_{r o c k} \rrbracket=W$ b.

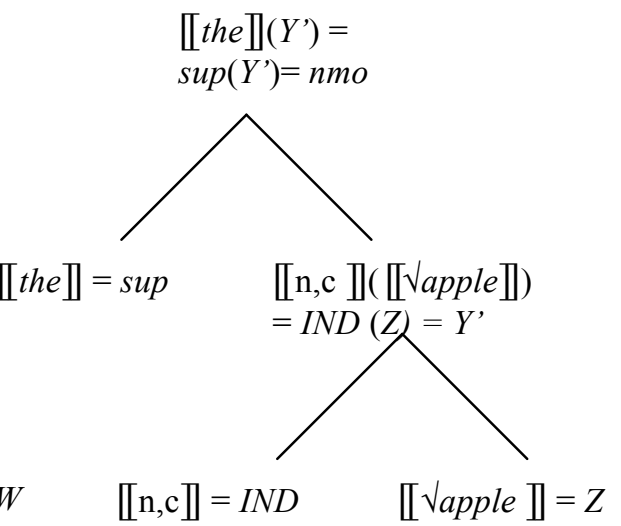


Figure 8: The root $\sqrt{ }$ rock denotes the non-individuated semi-lattice $W$ while the root $\sqrt{ }$ apple denotes the non-individuated semi-lattice $Z$. The function $I N D$ in this context maps $W$ to $X=\{a, b$, $c, a b, a c, b c, a b c\}$ which is an individuated semi-lattice. Also, the function $I N D$ in this context maps $Z$ to $Y^{\prime}=\{n, m, o, n m, n o, m o, n m o\}$ which is also an individuated semi-lattice. (a) shows the compositional meaning of determiner phrase the rocks. (b) shows the compositional meaning of

the determiner phrase the apples.

The function IND maps $W$ to a lattice that contains all of the rocks in the room as individuals while it maps $Z$ to a lattice that contains all the apples in the room as individuals. The determiner the then selects the supremum of this lattice.

The interpretation of the singular morpheme is not crucial to our account, since only mass nouns and plural count nouns can appear in comparative constructions of the kind under consideration. Still, for completeness we provide an interpretation for the singular count noun head. Like Link (1983) and Chierchia (1998), we propose that singular nouns denote only a set of individuals without any higher aggregates. To do this we exploit the IND function as follows:

Interpretation of the singular head: $\llbracket \mathrm{n}, \mathrm{c}, \mathrm{sg} \rrbracket=\lambda \mathrm{P}(\{\mathrm{x}: \mathrm{x}$ is an individual in $\operatorname{IND}(\mathrm{P})\})$

Thus, singular nouns have denotations that only contain the atoms of the count noun denotation.

\subsection{An account of generalizations 1 and 2}

In this section, we describe how our proposal explains the comparative data (Section 5.3.1), and thus how it explains the two generalizations in Section 4 (Section 5.3.2).

\subsubsection{Truth conditions for comparatives}

To account for the truth conditions of comparative sentences we must first outline a plausible interpretation for such sentences. For simplicity, we assume that more is a determiner that takes sets as arguments. Also, we assume that more can syntactically combine with either count or mass noun phrases (although not with singular count nouns). Furthermore, we will ignore issues concerning ellipsis in than clauses (see Kennedy, 1999; Hoeksema, 1983; Bresnan, 1975; Lechner, 1999), as well as quantifier movement (see Hackl, 2000; Heim, 2000; Kennedy 1999; Larson, 1988), and general concerns with respect to compositionality (see Cresswell, 1976; 
Kennedy, 1999; Larson 1988; von Stechow 1984). These issues are important and can be empirically addressed; however, we will focus on the semantics of the noun phrase rather than other aspects of comparative semantics. What we outline is not a compositional semantics for comparatives sentences, but rather the truth conditions that reflect speaker intuitions. To do so, we will take the sentence schema in (23) as our basic sentence structure.

(23) Y has more NP than Z has.

Whatever the correct syntactic and compositional structure is for sentences that follow the schema in (23), we assume that the truth conditions are based upon a comparison of two sets: the set of things/stuff that are in the denotation of the NP and that are possessed by Y compared to the set of things/stuff that are in the denotation of the NP that are possessed by $\mathrm{Z}$. The interpretation of more specifies how these two sets are compared.

\section{Truth conditions for sentences that fit the schema in (23):}

$\llbracket \mathrm{Y}$ has more NP than $\mathrm{Z}$ does $\rrbracket=\llbracket$ more $\rrbracket(\{\mathrm{x}:$ owns $\llbracket \mathrm{Y} \rrbracket, \mathrm{x}) \& \llbracket \mathrm{NP} \rrbracket(\mathrm{x})\},\{\mathrm{x}:$ owns $(\llbracket \mathrm{Z} \rrbracket$, $\mathrm{x}) \& \llbracket \mathrm{NP} \rrbracket(\mathrm{x})\})$

Note that the two arguments of more are subsets of the denotation of the NP. In fact, the arguments are themselves join semi-lattices. Possession is closed under sums (if Y owns $x$ and Y owns $z$, then Y possesses $x \oplus z$ ) and possession is closed under the lattice part-of relation (if Y owns $x \in \llbracket \mathrm{NP} \rrbracket$, then for any $z \leq x$ such that $z \in \llbracket \mathrm{NP} \rrbracket$, Y owns $z$ ). It follows from these two facts that if the NP denotation is an individuated semi-lattice then both arguments to more are also individuated semi-lattices. Similarly, if the NP denotation is a non-individuated semi-lattice then both arguments to more are also non-individuated. Such consequences are crucial for our account.

Given that more takes two semi-lattices as arguments, we define its semantics as follows. ${ }^{19}$

\footnotetext{
${ }^{19}$ For simplicity we present a non-conservative semantics for more. However, conservativity can be maintained if more is viewed as a three-place quantifier with the entire domain covertly serving as the third argument. See Keenan \& Westerstahl (1997) for arguments and a discussion. Alternatively, more could be viewed as a modifier of a covert adjective, in which case it would not be subject to conservativity.
} 
Interpretation of the determiner more: $\llbracket$ more $\rrbracket=\lambda X \lambda Y[\mu(\mathrm{X}, \mathrm{Y})]$

Contextual conditioning of measure function $\mu$ : $\mu$ is interpreted as one of the setcomparison functions $m_{z}$ in the series $\left\langle m_{1}, m_{2}, m_{3}, m_{4} \ldots m_{n}\right\rangle$ such that the argument for $\mu$ is in the range of $m_{z}$. Furthermore, contextually $m_{z}$ is preferred to $m_{y}$ if $z<y$.

We make the comparison with more context-dependent since the comparison is different for different types of NPs. Comparison is based on number for furniture and apples, on weight or volume for water and mud, and on emotional intensity for anger and fear. As the type of denotation for the NP changes, the set-comparison functions in $\mu$ also change. For now, let's concentrate on comparisons by number. We will specify this comparison function to be the first in the series. It is the default comparison.

Definition of the measure function $\boldsymbol{m}_{1}: m_{1}$ is defined only for sets of aggregates that are individuated semi-lattices. When defined, $m_{l}(X, Y)=1$ iff $X$ and $Y$ are join semi-lattices and $\mid\{x: x$ is an individual in $X\}|>|\{y: y$ is an individual in $Y\} \mid$.

The function $m_{l}$ compares the number of individuals in each semi-lattice, where individuals are defined as above. According to our definition of $m_{l}$, if the first argument has more individuals than the second, then the comparative sentence is true. Otherwise the comparative sentence is false. Note that $m_{1}$ might not be the only function that makes use of the set of individuals in a lattice. Interpretations for other quantifiers such as two might also rely on defining a set of individuals. However, other quantifiers, such as all, do not require such a set. We give possible interpretations for such quantifiers below.

$\llbracket$ two $\rrbracket=\lambda X \lambda Y(\mid(\{x: x$ is an individual in $X\} \cap Y) \mid \geq 2$

$\llbracket$ all》 $=\lambda X \lambda Y(\{z: z \in X \cap \operatorname{Cov}\} \subseteq Y)$, where $\operatorname{Cov}$ is a contextually defined cover as discussed in Gillon (1992) and Schwarzschild (1996). 
According to our truth-conditions, comparisons of number operate on lattice-theoretically defined individuals in two given sets. This means that more can only compare by number if its NP argument is interpreted as an individuated semi-lattice. Non-individuated semi-lattices must use another type of comparison, such as a comparison by weight. We will forego the details of how such a comparison is done for now, but we will assume that the comparison function $m_{2}$ makes such a comparison.

This proposal for implementing truth conditions differs from the proposals advanced by Klein (1981), Hackl (2000), and Cresswell (1976). These authors either do not address the multiple ways of comparing nominals or their proposals are incompatible with the latticetheoretical approach explored in this paper. Still, extensionally speaking, the truth conditions presented above overlap significantly (if not completely) with all of these previous proposals.

Given the truth-conditions established thus far, let us consider examples like those mentioned in Section 3.

(24) a. Seymour has more apples than Esme has.

b. Seymour has more apple than Esme has.

c. Seymour has more furniture than Esme has.

According to our semantics, the truth of (24a) and (24c) depend on a comparison of the number of individuals that Seymour and Esme each own. The truth conditions of (24a) are given in (25).

$\llbracket$ more $\rrbracket(\{x:$ owns $(\llbracket$ Seymour $\rrbracket, x) \& \llbracket$ apples $\rrbracket(x)\}$, $\{\mathrm{x}: \operatorname{owns}(\llbracket$ Esme $\rrbracket, \mathrm{x}) \& \llbracket \operatorname{apples} \rrbracket(\mathrm{x})\})$ $=m_{l}(\{\mathrm{x}: \operatorname{owns}(\llbracket$ Seymour $\rrbracket \mathrm{x}) \& \llbracket \operatorname{apples} \rrbracket(\mathrm{x})\}$, $\{\mathrm{x}: \operatorname{owns}(\llbracket$ Esme $\mathbb{b} \mathrm{x}) \& \llbracket \operatorname{apples} \rrbracket(\mathrm{x})\})$ $=\mid\{x: x$ is an individual in $\{\mathrm{x}:$ owns $(\llbracket$ Seymour $\rrbracket, \mathrm{x}) \& \llbracket \operatorname{apples} \rrbracket(\mathrm{x})\}\} \mid$ $>\mid\{y: y$ is an individual in $\{\mathrm{x}:$ owns $(\llbracket$ Esme $\rrbracket, \mathrm{x}) \& \llbracket$ apples $\rrbracket(\mathrm{x})\}\} \mid$

Since apples is a count-noun, its denotation must be an individuated semi-lattice (according to the function $I N D$ ). As a result, the two sets that serve as arguments for more are also individuated semi-lattices. This means that the two sets can be compared via the function $m_{l}$. 
The result is that the comparison is true if the number of apples that Seymour has is greater than the number that Esme has.

A similar analysis can be given for the sentence in (24c). For example, consider the truth conditions in (26).

$$
\begin{aligned}
& \llbracket \text { more } \rrbracket(\{\mathrm{x}: \text { owns }(\llbracket \text { Seymour } \rrbracket, \mathrm{x}) \& \llbracket \text { furniture } \rrbracket(\mathrm{x})\}, \\
& \quad\{\mathrm{x}: \text { owns }(\llbracket \text { Esme } \rrbracket, \mathrm{x}) \& \llbracket \text { furniture }]](\mathrm{x})\}) \\
& =m_{I}(\{\mathrm{x}: \text { owns }(\llbracket \text { Seymour } \rrbracket \mathrm{x}) \& \llbracket \text { furniture } \rrbracket(\mathrm{x})\} \\
& \quad\{\mathrm{x}: \text { owns }(\llbracket \text { Esme } \rrbracket, \mathrm{x}) \& \llbracket \text { furniture } \rrbracket(\mathrm{x})\}) \\
& =\mid\{x: \mathrm{x} \text { is an individual in }\{\mathrm{x}: \text { owns }(\llbracket \text { Seymour } \rrbracket, \mathrm{x}) \& \llbracket \text { furniture } \rrbracket(\mathrm{x})\}\} \mid \\
& \quad>\mid\{y: y \text { is an individual in }\{\mathrm{x}: \text { owns }(\llbracket \text { Esme } \rrbracket, \mathrm{x}) \& \llbracket \text { furniture } \rrbracket(\mathrm{x})\}\} \mid
\end{aligned}
$$

The only difference between (25) and (26) is that furniture is a mass noun. As a mass noun, it has the same denotation as the root lexical item $\sqrt{ }$ furniture. However, this lexical item has an individuated semi-lattice as its denotation, unlike Vapple. As a result, the sets compared by more are individuated semi-lattices and hence the truth of the sentence relies on comparing the number of pieces of furniture owned by Seymour to the number of pieces owned by Esme.

The truth conditions change dramatically for sentences like the one in (24b). The truth conditions for (24b) are presented in (27).

$$
\begin{aligned}
& \llbracket \text { more } \rrbracket(\{x: \text { owns }(\llbracket \text { Seymour } \rrbracket, x) \& \llbracket \text { apple } \rrbracket(x)\}, \\
& \{\mathrm{x}: \operatorname{owns}(\llbracket \text { Esme } \rrbracket, x) \& \llbracket \text { apple } \rrbracket(x)\}) \\
& =m_{2}(\{\mathrm{x}: \operatorname{owns}(\llbracket \text { Seymour } \rrbracket, \mathrm{x}) \& \llbracket \text { apple } \rrbracket(\mathrm{x})\} \text {, } \\
& \{\mathrm{x}: \text { owns }(\llbracket \text { Esme } \rrbracket, x) \& \llbracket \text { apple } \rrbracket(x)\})
\end{aligned}
$$

Unlike in (24a), the NP complement of more is a mass noun. Unlike in (24c), the mass noun does not denote an individuated semi-lattice. If it did then the root could not appear in a count noun phrase as it does in (24a). As a result, the two sets compared by more are non-individuated semilattices, and cannot be compared by the function $m_{l}$. The next applicable function in $\mu$ must be used instead, such as the function $m_{2}$, which computes a comparison by weight. 
In summary, by exploiting the various options given for the noun phrase semantics provided in Section 5.2 it is possible to explain the different types of judgments for comparative sentences involving bare NP arguments.

\subsubsection{Two generalizations explained}

Given the interpretation of functional heads presented here, the two generalizations in Section 3 can be explained. In Generalization 2 we noted that some mass nouns denote individuals while others do not. As demonstrated by our discussion of comparative constructions, denotations that contain individuals allow for assessments or measurements based on number, whereas denotations without individuals do not. Thus, according to our proposal, Generalization 2 follows from the fact that lexical roots can be mapped either to individuated lattices (as in the case of $\sqrt{\text { furniture }}$ ) or to non-individuated lattices (as in the case of $V_{\text {water }}$ and $\sqrt{\text { apple). }}$. Since the mass noun functional head is interpreted as an identity function, the resulting interpretation of mass nouns can be either individuated or non-individuated.

Generalization 1 states that any lexical item that can be used as a count noun cannot denote individuals when used as a mass noun. This follows from our interpretation of the count noun functional head. This functional head is interpreted as a function from non-individuated lattices to individuated lattices. Thus, not only do all count nouns necessarily have individuals as part of their denotation, but corresponding root lexical items cannot have individuals in their denotations. Since the interpretation of the mass noun is identical to the interpretation of the root lexical item, the mass noun counterpart to any count noun cannot have individuals as part of its denotation. A further consequence of this semantic interpretation is that lexical items that are

interpreted as individuated semi-lattices such as $\sqrt{ }$ furniture and $\sqrt{ }$ equipment can never appear as count nouns. This explains why *three furnitures and *three equipments are not acceptable count noun phrases.

\section{Concluding remarks}

We presented data from comparative constructions to investigate the semantic interpretation of mass and count nouns. The purpose of this investigation was to explore, via the case study of the 
mass-count distinction, how lexical and syntactic representations interact in the generation of phrase level interpretations.

Based on the data generated by comparative constructions, we established two empirical generalizations. First, we noted that nouns which can occur in either mass or count syntax undergo a shift in the dimension of comparison according to the syntactic context in which they are used. When used as count nouns, nouns specify comparison by number. When used as mass nouns, the very same nouns do not permit comparison by number. Second, we noted that, like count nouns, some mass nouns also specify comparison by number. To capture these two generalizations, we hypothesized that root nouns are not specified as mass or count. We argued that nouns are formed by combining roots with functional heads, and that root nouns can be associated with denotations that either do or do not contain individuals as minimal parts. The interpretation of the count noun head maps denotations that do not contain individuals to denotations that do. In contrast, the mass noun head is interpreted as an identity function. Thus, if the root noun is mapped to a denotation with individuals, then the mass noun version of this root will also be mapped to such a denotation. Also, since the count noun head only ranges over root nouns that are not associated with individuals, it follows that such nouns should never denote individuals when used in mass syntax.

We argued that existing theories have difficulty accounting for these facts because they fail to provide a grammatical account that explains the comparative data. Theories like Link's (1983), which posit separate semantic domains for mass and count nouns, do not distinquish between the subtypes of mass nouns, and assign all mass nouns a common type of denotation. Other theories, like those of Gillon (1999), Bunt (1985), and Chierchia (1998), which appeal to context or world knowledge to explain the interpretation of mass nouns, fail to explain why denotations of noun phrases change as mass-count syntax varies, when the contextual setting is constant. The fact that some mass nouns do not denote individuals even when they are present in a context (e.g., stone) indicates that comparative judgments require a grammatical explanation, rather than one that appeals to context or world knowledge. Thus, theories that posit a uniform mass semantics and theories that posit non-linguistic mechanisms for explaining their heterogeneity fail to account for the data presented here. These data require a theory that predicts heterogeneous mass noun interpretations and explains them via grammatical representations. The current paper provides one way in which these requirements might be fulfilled, and establishes 
the minimal requirements for future theories of the mass-count distinction.

Beyond the facts described here, the current proposal also has significant implications for the study of noun semantics cross-linguistically. Many languages feature comparative constructions akin to those in English, and thus permit their use for assessing mass and count interpretations. Languages vary considerably with respect to the frequency with which words are used as mass or count (if at all), and also with respect to which words denote individuals as mass nouns. As a result, analyzing comparative judgments cross-linguistically will provide the strongest test for the current proposal, and for any future theory as well. Our view predicts that languages with a mass-count distinction will exhibit the same generalizations as in English, regardless of item-by-item differences in how words are used. So long as a word can denote individuals as a mass noun, it should not appear also in count syntax. Also, there should be no distinction between the interpretations of root nouns and the interpretation of mass nouns. Thus, in languages that lack count syntax (e.g., Japanese, Chinese) nouns should be interpreted like mass nouns in English, and receive both individuated an unindividuated interpretations.

\section{Acknowledgements}

We would like to thank Brendan Gillon, Gennaro Chierchia, Jesse Snedeker, Kyle Johnson, Heather Newell, Dana Isac, Mark Hale, Charles Reiss, the Concordia LSA and an anonymous reviewer for helpful comments. This research would not have been possible without grants from the Social Sciences and Humanities Research Council of Canada, grant numbers 752-2001-1304 and 756-2006-0484.

\section{References}

Barner, D., \& Bale, A. (2002). No nouns, no verbs: psycholinguistic arguments in favor of lexical underspecification. Lingua, 112: 771-791.

Barner, D., \& Bale, A. (2005). No nouns, no verbs? Rejoinder to Panagiotidis. Lingua, 115: $1169-1179$.

Barner, D., \& McKeown, R. (2005). The syntactic encoding of individuation in language and language acquisition. In A. Brugos, M. R. Clark-Cotton, \& S. Ha (Eds.), BUCLD 28: Proceedings of the 28th annual Boston University conference on language development. Cascadilla. Somerville. 61-72.

Barner, D., \& Snedeker, J. (2005). Quantity judgments and individuation: Evidence that mass nouns count. Cognition, 97: 41-66.

Barner, D., \& Snedeker, J. (2006). Children's early understanding of mass-count syntax: individuation, lexical content, and the number asymmetry hypothesis. Language Learning and Development, 2: 163-194. 
Barner, D., \& Wagner, L., \& Snedeker, J. (2008). Events and the ontology of individuals: Verbs as a source of individuating mass and count nouns. Cognition, 106: 805-832.

Bauer, L. (1973). English word formation. Cambridge University Press. Cambridge.

Bloom, P. (1994). Semantic competence as an explanation for some transitions in language development. In Y. Levy (Ed.), Other children, other languages: Theoretical issues in language development. Lawrence Erlbaum Associates, Inc. Hillsdale. 41-76.

Bloom, P. (1999). The role of semantics in solving the bootstrapping problem. In R. Jackendoff, P. Bloom, \& K. Wynn (Eds.), Language, logic, and concepts: Essays in memory of John Macnamara. MIT Press. Cambridge. 285-310

Borer, H. (2005). Structuring sense, volume 1: In name only. Oxford University Press. Oxford.

Bresnan, J. (1975). Comparative deletion and constraints on transformations. Linguistic Analysis, 1(1): 25-75.

Bunt, H. C. (1979). Ensembles and the formal semantics properties of mass terms. In Pelletier, J. (Ed.), Mass terms: Philosophical problems. Synthese Language Library, 6. D. Reidel. Dordrecht. 249-277.

Bunt, H. C. (1985). Mass terms and model-theoretic semantics. Cambridge University Press. New York.

Chierchia, G. (1998). Plurality of mass nouns and the notion of 'semantic parameter'. Events and Grammar, 70: 53-103.

Chomsky, N. (1970). Remarks on nominalization. In Jacobs, R., Rosenbaum, P. (Eds.), Readings in english transformational grammar. Blaisdell Waltham. 184-221.

Clark, E.V. (1982). The young word-maker: A case study of innovation in the child's lexicon. In Wanner, E., Gleitman, L.R. (Eds.), Language acquisition: The state of the art. Cambridge University Press. Cambridge. 390-425.

Clark, E.V. (1993). The lexicon in acquisition. Cambridge University Press. Cambridge.

Corbin, D. (1997). Entre les mots possibles et les mots existants: les unite' s lexicales a' faible probablite' d'actualisation. In Corbin, D. (Ed.), Mots possibles et mots existants. Universite' de Lille, Silexicales 1. 79-90.

Cresswell, M. (1976). The semantics of degree. In B. Partee (Ed.), Montague grammar. Academic Press. New York. 261-292.

Dressler, W. U., Ladanyi, M. (2000). Productivity in word formation (WF): A morphological approach. Acta Linguistica Hungarica 47: 103-144.

Gathercole, V. (1985). More and more and more about more. Journal of Experimental Child Psychology, 40: 73-104.

Gillon, B. (1992). Towards a common semantics for English count and mass nouns. Linguistics and Philosophy, 15: 597-640.

Gillon, B. (1999). The lexical semantics of English count and mass nouns. In E. Viegas (Ed.), The breadth and depth of semantic lexicons. Kluwer. Dordrecht. 19-37

Gillon, B., E. Kehayia and V. Taler (1999). The mass count distinction: Evidence from psycholinguistic performance. Brain and Language, 68: 205-211.

Gordon, P. (1985). Evaluating the semantic categories hypothesis: The case of the mass/count distinction. Cognition, 20: 209-242.

Hackl, M. (2000). Comparative quantifiers. Ph. D. Thesis, MIT.

Halle, K. \& Marantz, A. (1993). Distributed morphology and the pieces of inflection. In Keyser, S.J., Hale, K. (Eds.), The view from building 20. MIT Press. Cambridge. 111-176. 
Harley, H. \& Noyer, R. (1999). Licensing in the non-lexicalist lexicon: Nominalization, roots and the encyclopedia. MIT working papers in linguistics 32:119-137.

Heim, I. (2000). Degree operators and scope. In Proceedings of SALT X. CLC Publications. Cornell. 40-64.

Hoeksema, J. (1983). Negative polarity and the comparative. Natural Language and Linguistic Theory, 1: 403-434.

Jackendoff, R. (1991). Parts and boundaries. Cognition, 41: 9-45.

Keenan, E.L. \& Westerstahl, D. (1997). Generalized quantifiers in linguistics and logic. In van Benthem, J. \& ter Meulen, A. (eds.), Handbook of logic and language. Elsevier. Amsterdam. 837-93.

Kennedy, C. (1999). Projecting the adjective: The syntax and semantics of gradability and comparison. Garland. New York

Klein, E. (1981). The interpretation of adjectival, nominal, and adverbial comparatives, In Groenendijk, J.; Janssen, T.; and Stokhof, M. (eds.), Formal methods in the study of language. Mathematical Center Tracts. Amsterdam.

Krifka, M. (1995). Common nouns: A contrastive analysis of Chinese and English. In G. Carlson \& F. Pelletier (Eds.), The generic book. University of Chicago Press. Chicago. 398-411

Langendoen, T. (1978). The logic of reciprocity. Linguistic Inquiry 9(2): 177-197.

Larson, R. (1988). Scope and comparatives. Linguistics and Philosophy 11: 1-26.

Lechner, W. (1999). Comparatives and DP-structure. Ph.D. dissertation, University of Massachusetts, Amherst.

Link, G. (1983). The logical analysis of plurals and mass terms: A lattice-theoretical approach. In R. Bauerle, C. Schwarze, \& A. Stechow (Eds.), Meaning, use, and interpretation of language. de Gruyter. Berlin. 302-323

Link, G. (1998). Algebraic semantics in language and philosophy. Center for the Study of Language and Information. Stanford.

Macnamara, J. (1986). A border dispute: The place of logic in psychology. MIT Press. Cambridge.

Marantz, A. 1997. No escape from syntax: don't try morphological analysis in the privacy of your own lexicon. University of pennsylvania working papers in linguistics. 4: 201-225.

McCawley, J. D. (1979). Lexicography and the count-mass distinction. In J. D. McCawley. Adverbs, vowels, and other objects of wonder. University of Chicago Press. Chicago. 165173.

Pesetsky, D. (1994). Zero syntax: Experiencers and cascades. MIT Press. Cambridge.

Quine, W. V. O. (1960). Word and object. MIT Press. Cambridge.

Rainer, F. (1988). Towards a theory of blocking. In Booji, G., van Marle, J. (Eds.), Yearbook of Morphology. Kluwer. Dordrecht. 155-185.

Sharvey, R. (1979). The indeterminacy of mass predication. In Pelletier, J. (Ed.), Mass terms: Philosophical problems. Synthese Language Library, 6. D. Reidel. Dordrecht. 47-54.

Schwarzschild, R. (1996). Pluralities. Studies in the linguistics and philosophy 61. Kluwer. Dordrecht.

von Stechow, A. (1984). Comparing semantic theories of comparison. Journal of Semantics, 3: $1-77$.

Wisniewski, E. J., Imai, M., \& Casey, L. (1996). On the equivalence of superordinate concepts. Cognition, 60: 269-298. 


\author{
Alan C. Bale \\ Concordia University, H663 \\ 1455 de Maisonneuve Blvd., W. \\ Montréal, QC (H3G 1M8) \\ Canada \\ alancbale@gmail.com \\ David Barner \\ Department of Psychology \\ University of California, San Diego \\ 5336 McGill Hall \\ 9500 Gilman Drive \\ La Jolla, CA 92093-010 \\ barner@ucsd.edu
}

